\title{
Light-induced Phase Transition of Lyotropic Liquid Crystals and Motion Behavior of Micron Objects via Photochemical Azobenzene Molecules
}

\author{
Su MA ${ }^{1, a}$, Yutaka KUWAHARA ${ }^{1,2}$, Tomonari OGATA ${ }^{3}$, Sunnam $\mathrm{KIM}^{1}$, \\ Seiji KURIHARA ${ }^{1,2,3,4, b}$
}

${ }^{1}$ Department of Applied Chemistry and Biochemistry, Graduate School of Science and Technology, Kumamoto University, 2-39-1 Kurokami, Chuo-ku, Kumamoto, 860-8555, Japan

${ }^{2}$ Kumamoto Institute for Photo-Electro Organics (PHEONICS), 3-11-38 Higashimachi, Higashi-ku, Kumamoto, 862-0901, Japan

${ }^{3}$ Innovative Collaboration Organization, Kumamoto University, 2-39-1 Kurokami, Chuo-ku, Kumamoto, 860-8555, Japan

${ }^{4}$ JST-CREST, K's Gobancho, 7 Gobancho, Chiyoda-ku, Tokyo, 102-0076, Japan

amasu115@hotmail.com, bkurihara@kumamoto-u.ac.jp

Keywords: Lyotropic liquid crystal, Phase transition, Azobenzene, Motion behavior.

\begin{abstract}
Ternary lyotropic liquid crystal (LLC), was prepared by mixing tetradecyltrimethylamonium bromide (MTAB), water and azobenzene (Az). The phase transition of the LLC was induced from LC phase to isotropic (I) one upon Ar+ laser, which was owing to the trans-cis-trans phoisomerizaion cycle of Az during the irradiation. After that, the LC phase gradually recovered from I phase to nematic $(\mathrm{N})$ phase when being exposed to dark condition. It was also suggested that the recovery time from I to $\mathrm{N}$ was different with the Az structures, temperature and ratio of $\mathrm{MTAB} / \mathrm{Az}$ in the ternary system. Then, the effect of light irradiation on the motion behavior of micron objects dispersed in the ternary LLC film was studied. It was found that the micron objects which dispersed in the irradiated area moved toward the center of the irradiated area, while the micron objects which dispersed around the irradiated area moved away from the irradiated area.
\end{abstract}

\section{Introduction}

Az can exhibit trans-cis isomerization by UV and reversible one by visible (Vis) light. Owing to the photo-isomerization behavior, much effort has been focused on the Az compounds to control phase transition of LCs as well as alignment of LCs for applications on optical displays and assembly technology, which have been studied by various external stimuli, such as gravitational field, external electric, magnetic fields, thermo, and light [1-8].

Many works have recorded the application on assembly technology and manipulation of micron objects, and the driving force is ascribed to surface tension gradient or phase transition. As the paper reported by S.N. Varanakkottu, et al, the inward flow are induced when Az compound are dissolved in water and irradiated with UV light, which is supported by the gradient in surface tension between the difference of trans-form and cis-form Az [9]. Similarly, the gradient in surface tension can also be used to control the motion of glass rods in Az-doped polyethylene glycol films, as found by Su Ma et al [10]. However, Lohn, et al described the micron objects can be assembled by thermos-induced phase transition [2], whose driving force is also accorded with our previous work [6-7, 12].

In addition, LLC phases, as one type of LCs, are abundant in living systems. For example, many proteins and cell membranes are LLCs. Other well-known examples of LLCs are solutions of soap and various related detergents, as well as the tobacco mosaic virus [13-15]. So the LLC also plays an important role in our daily life, while there is little or no related study reported on the manipulation of micron objects on the surface of LLCs. Herein, we investigated the effect of hydrophilic Az compounds, A3 and HA3 (the molecular structures were shown in Scheme1), on the phase behavior of LLC and a manipulation of micron objects (glass rods) on Az-doped LLC films. 


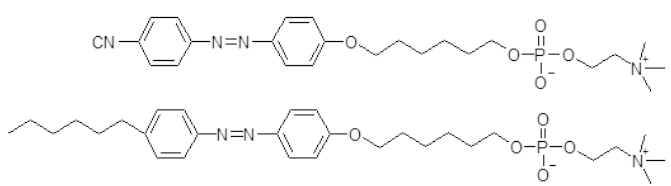

Scheme 1. Structures of Azobenzene-Containing compound A3 and HA3

\section{Experiment}

The photoisomerization behaviour of A3 and HA3, synthesized by literature methods [16], were examined in methanol solution by monitoring changes in the absorption spectra from a UV/Vis spectrometer at room temperature. Then, the samples of ternary LLCs, containing tetradecyltrimethylamonium bromide (MTAB, 39.6\%), water (60.4\%) and Az (A3/HA3), which was prepared according to the procedure described in reference [17], were prepared. To reduce water evaporation losses from the system, the Az-doped or together with micron objects-including LLC films were sealed up using UV-curable adhesive.

\section{Results and Discussion}

Photoisomerizaiton Behavior. As shown in Fig. 1, for A3-methanol solution, the UV/Vis absorption spectra were characterized by a strong $\pi-\pi^{*}$ transition of trans-A3 moiety at about $360 \mathrm{~nm}$ and a weak absorption at about $440 \mathrm{~nm}$, which originated from $\mathrm{n}-\pi^{*}$ transition. The absorbance decreased at about $360 \mathrm{~nm}$ and increased at $440 \mathrm{~nm}$ when the solution was exposed to UV ( $\lambda=365 \mathrm{~nm}$, $30 \mathrm{~mW}$ ), indicating the photoisomeriztion from the trans-form to the cis-form occurred. Similarly, HA3 also exhibited the same photoisomerization behavior as A3 in methanol solution, whose typical trans-/cis-isomer was assigned to the peaks at $350 \mathrm{~nm}$ and $440 \mathrm{~nm}$, as shown in Fig.1(c).
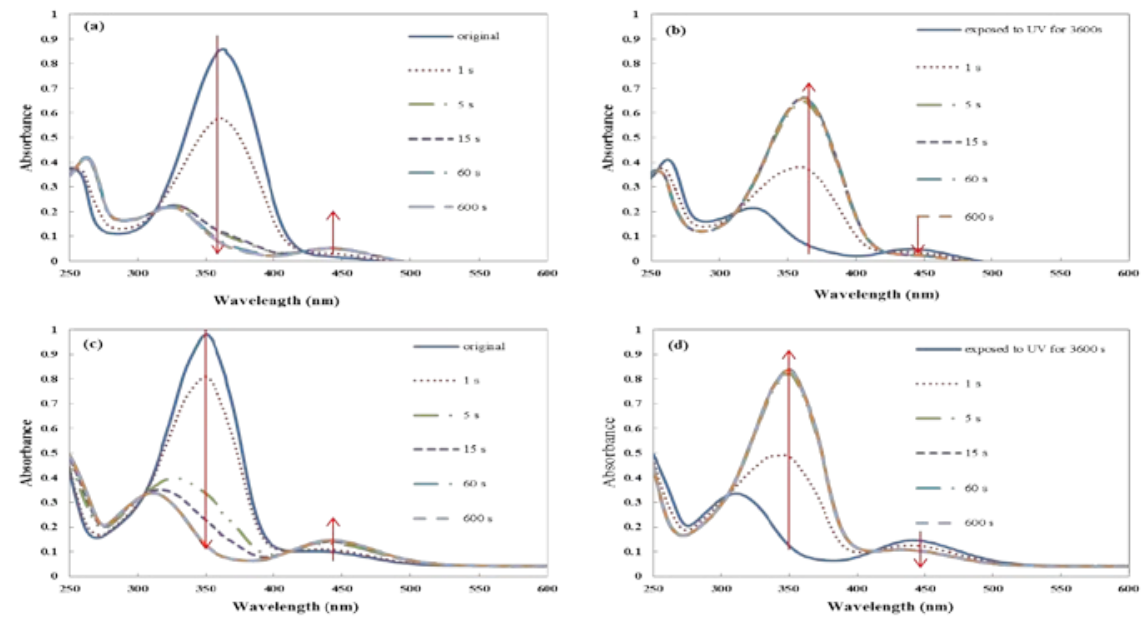

Fig. 1 Changes in absorption spectra of A3 (a) (b) and HA3(c) (d) in methanol solution by irradiation with UV (a) (c) and Vis light (b) (d) at room temperature

The photostatioary state reached after $60 \mathrm{~s}$ for both of A3-methanol and HA3-methanol solution. In addition, Vis light $(\lambda=436 \mathrm{~nm}, 130 \mathrm{~mW})$ irradiation caused an increase and a decreased of the absorbance values at about $360 \mathrm{~nm}$ and $440 \mathrm{~nm}$, indicating the back photoisomerization from cis to trans be induced upon Vis light. The trans-cis isomerization rate of A3 and HA3 was calculated as $92.0 \%$ and $87.2 \%$, respectively when exposed to UV light for $600 \mathrm{~s}$. The cis-trans isomerization rate was also different and gave a rate $48.1 \%, 37.7 \%$ within $1 \mathrm{~s}$ upon Vis light, which suggested that the cis-A3 was more easily to isomerize into trans form compared to the cis-HA3. This was maybe owing to that comparatively strong electron withdrawing substituents - $\mathrm{CN}$ in $\mathrm{A} 3$ decrease the barrier for isomerization, thus A3 underwent faster cis-trans isomerization compared to HA3 with electron donating substituent $-\mathrm{C}_{6} \mathrm{H}_{13}[18]$. 
Phase Transition of LLC by Thermal Effect. To explain the phase transition behavior of the LLCs with different Az compounds and different ratio of MTAB/Az, POM and DSC was carried out, respectively. Fig.2 (a), (b) and (c) exhibited the typical $\mathrm{N}$ textures, which were associated with samples MTAB, MTAB: A3=95:5 and MTAB: A3=98:2. In addition, as shown in Fig.2 (d) (e) and (f), all the 3 samples showed one endothermic peak ranging from $20{ }^{\circ} \mathrm{C}$ to $50{ }^{\circ} \mathrm{C}$. The results revealed that all samples, MTAB, MTAB: A3=95:5 and MTAB: A3=98:2, showed N as LC phase, and the phase transition temperature from $\mathrm{N}$ to I phase was almost same at about $36{ }^{\circ} \mathrm{C}, 36.5^{\circ} \mathrm{C}$ and $37^{\circ} \mathrm{C}$, respectively, indicating the addition of $\mathrm{A} 3$ had almost no effect on the phase behaviour of MTAB molecules in water.

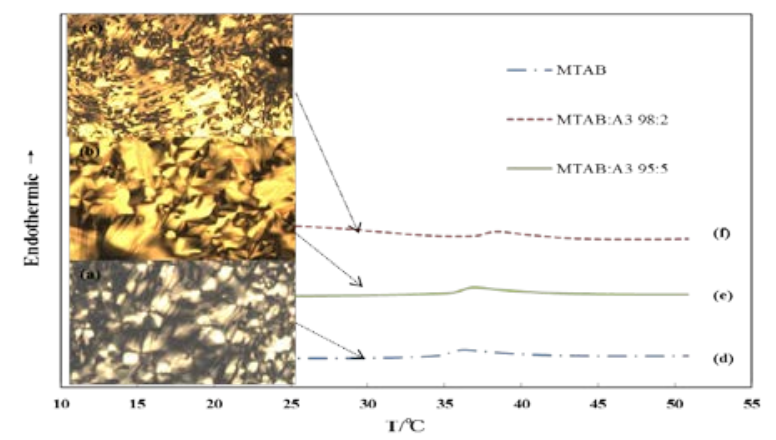

Fig. 2 Typical textures of MTAB-water LLCs and DSC heating thermograms of LLCs with different ratio of $\mathrm{MTAB} / \mathrm{Az}$ (a) (d) MTAB; (b) (e) MTAB: A3=95:5; (c) (f) MTAB: A3=98:2 (molar ratio)

The MTAB molecules doped with different amount of HA3 (MTAB: HA3=95:5/98:2) also exhibited almost the same N-I phase transition. However, compared to A3-doped LLC, the HA3-doped-LLC exhibited much higher phase transition temperature. For examples, the phase transition of MTAB: A3 $=95: 5$ from LC phase to I phase was about $37^{\circ} \mathrm{C}$, which was lower than that of $56^{\circ} \mathrm{C}$ for the sample of HA3 doped one. It is considered to contribute the fact that the trans-form HA3, doped in LLC, has a well ordered molecular arrangement with MTAB.
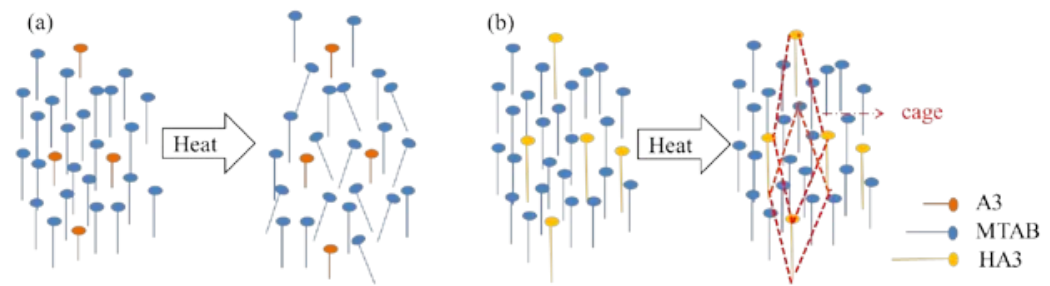

Fig. 3 Illustration of thermal-induced phase behavior for (a) A3-doped MTAB; (b) HA3-doped MAB

On the other hand, the molecular length of trans-HA3 is calculated as $21.1 \AA$ and relatively longer than that of MTAB (19.0 $\AA$ ), which also supports a "cage" or "frame" to keep the orientation of MTAB molecules in water. In contrast, trans-A3 (16.9 $\AA$ ) is shorter than that of MTAB, the "cage" can hardly be formed or invalid to fix the MTAB molecules in a limited a region to keep its well-ordered arrangement at high temperature, as the illustration in Fig.3. Therefore, the MTAB doped with HA3 LLC exhibited a higher phase transition temperature than that of A3.

Phase Transition of LLC by Vis Light. In addition to the thermal-induced phase transition, an irradiation with Ar+ laser, as an external stimulus, was also carried out to study its effect on phase behavior. During all the irradiation process, a thermostat was used to keep the samples at $30{ }^{\circ} \mathrm{C}$ which was less than the phase transition temperature. As shown in Fig.4, the none-Az-doped MTAB sample always exhibited the $\mathrm{N}$ phase, even though the sample was exposed to $\mathrm{Ar}+$ laser. The texture did not change at all, either. For the Az-doped samples, before irradiation, owing the molecules were arranged in order, all the two samples also showed the $\mathrm{N}$ phase. However, as shown in Fig.4 (b), when the Az-doped samples were irradiated with Ar+ laser, the samples lost their birefringence, so the phase transition from $\mathrm{N}$ to I state was induced. 


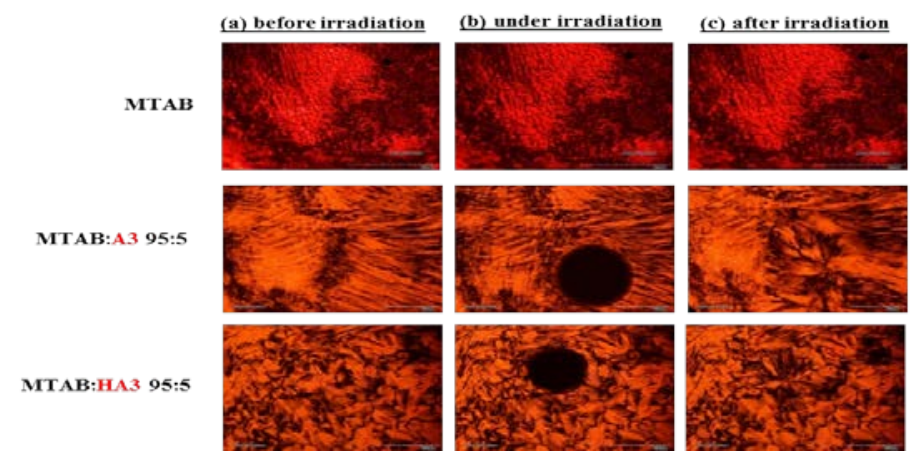

Fig. 4 Typical textures of MTAB-water LLC with different Az (MTAB/Az=95:5) (a) before; (b) under; (c) after irradiation using $\mathrm{Ar}+$ laser $\left(\mathrm{T}=30^{\circ} \mathrm{C}\right.$, light intensity=70 mW)

Similar experiment conducted in our previous work [6,7,12] explained the mechanism of phase transition by $\mathrm{Ar}+$ laser irradiation in terms of order-disrupting molecules $\mathrm{Az}$ through conformational changes. That means the phase transition can be induced in an Az-doped-LLC system in which Ar+ laser exposure directly decreased the order of the mixture through trans-cis-trans cycle photoisomerization of Az moiety. In addition, this photo-induced I state returned to the $\mathrm{N}$ phase (Fig. $4 \mathrm{c}$ ) spontaneously within a certain period of time after removal of the Ar+ laser. The surface textures of the samples after Ar+ laser irradiated were a little bit different from the one before irradiation, which was because of the thermostability for the trans-form $\mathrm{Az}$, thus the cis-trans isomerization occurred spontaneously along with the phase transition from I to $\mathrm{N}$ phase.

Effects of Az and Temperature on Recovery Time from I Phase to $\mathbf{N}$ Phase. Results from the photo-induced phase transition were suggested that trans-cis-trans cycle photoisomerization of A3 and HA3 was the driving force for the mechanism of phase transition behavior when being irradiated by $\mathrm{Ar}+$ laser. The responsive time of the phase transition from $\mathrm{N}$ to I was almost same (immediate) for all the samples. However, the recovery time from I to $\mathrm{N}$ varied in structures of Az, temperatures and $\mathrm{MTAB} / \mathrm{Az}$ ratios, as shown in Tab.1.

Tab.1 Effects of Az and temperature on recovery time (irradiated with Ar+ laser at $70 \mathrm{~mW}$ )

\begin{tabular}{ccccccc}
\hline No. & Az & $\begin{array}{c}\text { Molar ratio } \\
\text { (MTAB/Az })\end{array}$ & $\begin{array}{c}\text { Phase } \\
\text { transition }\end{array}$ & $\begin{array}{c}\text { Irradiatio } \\
\mathrm{n} \text { time[s] }\end{array}$ & $\begin{array}{c}\text { Recovery } \\
\text { time[s] }\end{array}$ & $\begin{array}{c}\text { Temperature } \\
{\left[{ }^{\circ} \mathrm{C}\right]}\end{array}$ \\
\hline 1 & No & & unavailable & 84 & & 30 \\
2 & A3 & $10: 1$ & available & 84 & 37 & 30 \\
3 & A3 & $95: 5$ & available & 84 & 107 & 30 \\
4 & A3 & $98: 2$ & available & 84 & 179 & 30 \\
5 & HA3 & $95: 5$ & available & 84 & $\approx 180$ & 30 \\
6 & HA3 & $95: 5$ & available & 84 & $\approx 300$ & 20 \\
7 & HA3 & $98: 2$ & available & 84 & $\approx 1080$ & 30 \\
8 & HA3 & $98: 2$ & available & 84 & $\approx 1800$ & 20 \\
\hline
\end{tabular}

To illustrate the importance of chemical structures of Azs those doped in LLC films, A3 and HA3 were used, which had a strong withdrawing substituent $-\mathrm{CN}$ and electron donating substituent $-\mathrm{C}_{6} \mathrm{H}_{13}$, respectively. Moreover, the two Az compounds also had different rigidity for the hydrophobic chain. After continuous $84 \mathrm{~s}$ exposure to $488 \mathrm{~nm}$ laser following exposure to dark condition, the recovery time of A3-doped-LLC film (No.3) from I state to $\mathrm{N}$ phase was much shorter than that of HA3-doped-LLC film (No.5). The same experimental phenomena was also found when compared No.4 to No.7. It was suggested that recovery time was shorter for A3 with a strong withdrawing substituent. One of the reasons was owing to the faster cis-trans isomerization of A3 molecules as the explanation in Fig.1. The other reason was considered to be the possibility that the HA3 doped LLCs had a flexible hydrophobic chain, such flexible chain drove HA3 to be more hindered and difficult to be completely recover from cis to trans. 
Additionally, it also can found out that with the ratio of the water-resolvable Az increased, the recovery time from I to $\mathrm{N}$ phase accelerated. The effect of temperature on recovery time was further illustrated between No.5 and No.6 in the table. The recovery time of HA3-doped-LLC film (No.5) which was exposed to $30{ }^{\circ} \mathrm{C}$ was much less than that to $20^{\circ} \mathrm{C}$ (No.6). The same experimental phenomena was also found when compared No.7 to No.8, which was considered to be the thermal motion of LLC molecules.

Motion Behavior of Micron Objects in LLC Film. In our previous work [12], we have demonstrated that the phase transition can support a driving force for the motion of micron objects. The driving force makes all micron objects move as a track of circles in the four cross regions which are divided by rubbing direction of the substrate and perpendicular line. Herein, the different motion behaviors were observed for the micron glass rods (G.R.) in A3-LLC (98:2) films. As shown in Fig.5, the G.R. dispersed in the irradiated area moved toward the center of isotropic area when being irradiated by Ar+ laser, whose motion behavior was totally different from the G.R. dispersed around irradiated area. That means the G.R. marked in ellipse in Fig.5 would move away from the dark area upon $\mathrm{Ar}+$ laser.

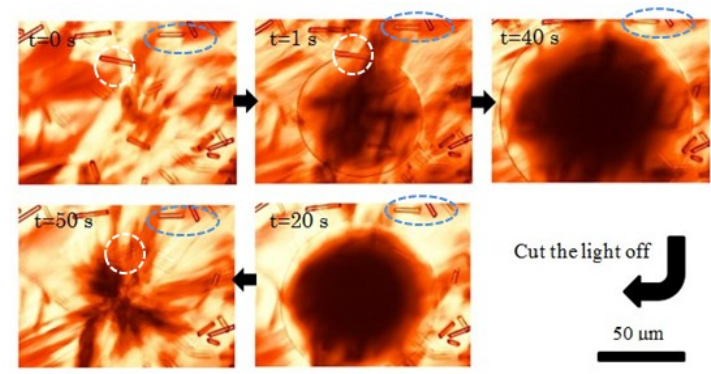

Fig.5 Optical micrographs of the G.R./A3/MTAB/ $\mathrm{H}_{2} \mathrm{O}$ (2:98) mixture irradiation with Ar+ laser (488 $\mathrm{nm}, 40 \mathrm{~mW}$ ) at $25^{\circ} \mathrm{C}$ in the glass cell (thickness: $200 \mu \mathrm{m}$ ). The term $\mathrm{t}$ in figures indicates the exposure time

In addition, the moved glass rods dispersed around the irradiated area could almost move back to the original position when being exposed to dark condition for some time, whose reversible motion behavior was not available for the glass rods dispersed in the irradiated area. According to our previous work $[8,9,12]$, it was suggested that the phase transition supported the main driving force for the motion behavior of glass rods placed around the irradiated area.
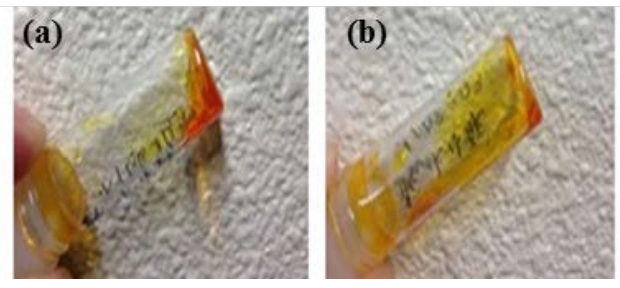

Fig. 6 Photographs of vials containing A3/MTAB/ $\mathrm{H}_{2} \mathrm{O}$ (2:98) LC molecules before (a) and after (b) irradiation with UV light for about $1 \mathrm{~min}$

Since monodirectional cis-trans isomerization can be induced by UV light, irradiation with UV light was carried out to investigate the viscosity changes. As shown in Fig.6 (a), before irradiation, the sample seemed to be a gel with high viscosity, while the sample turned into a state with low viscosity after irradiated by UV light as shown in Fig. 6 (b), because the sample flowed from the vial bottom to top. Thus, for the glass rods placed in the irradiated area, the surface tension gradient was considered to be the main driving force [9-10], when the LLC turned into a liquid state with low viscosity.

\section{Summary}

We have demonstrated that the HA3 molecules doped in LLCs are effective to preserve the molecular orientation of LLCs at relatively higher temperature, which means HA3 allowes the thermally 
induced a higher phase transition temperature from LC phase to I phase. It is also shown that the transition from LC phase to I one for the Az-doped LLCs films can also be induced by Vis light (Ar+ laser), the reversible phase transition of which can also be induced by exposure in dark condition. The recovery time of the reversible phase transition depends on the structure of Az molecule, temperature and ratio of MTAB/Az. The use of fixing Ar+ laser enables the control of micron glass rods in a way of moving toward and away the irradiation region for the ones dispersed in and around the irradiation region, whose driving force is due to the surface tension gradient and phase transition, respectively.

\section{Acknowledgements}

The present study was supported by CREST from JST, Japan. This work was also performed under the Cooperative Research Program of "Network Joint Research Center for Materials and Devices". The authors thank Prof. A. Muramatsu and Dr. K. Kanie from Tohoku University for synthetic help of the Az.

\section{References}

[1] A.B.G.M. Leferink op Reinink, E. van den Pol, V.A. Petukhov, G.J. Vroege and H.N.W. Lekkerkerker: Eur. Phys. J. Special Topics 222 (2013), 3053.

[2] L.L. West, A. Glushchenko, G. Liao, Y.R.D. Andrienko, and M.P: Phys. Rev. E 012702 (2002), 66.

[3] S. Lee, S. Oh, J. Lee, Y. Malpani, etc: Langmuir 29 (2013), 5869.

[4] S.N. Kim, T. Nakamura, R. Yagi, Y. Kuwahara, T. Ogata, S. Ujiie and S. Kurihara: Polymer International 63 (2013), 733.

[5] M. Kamruzzaman, S.N. Kim, Y. Kuwahara, T. Ogata, S. Kurihara: O. J. P. Chem. 3 (2013), 92.

[6] A. Kausar, H. Nagano, T. Ogata, T. Nonaka, and S. Kurihara: Angew. Chem. Int. Ed. 48 (2009), 2144.

[7] A. Kausar, H. Nagano, Y. Kuwahara, T. Ogata, and S. Kurihara: Chem. Eur. J. 17 (2011), 508.

[8] A. Diguet, R.M. Guillermic, N. Magome, A.S. Jalmes, Y. Chen, K. Yoshikawa, and D. Baigl: Angew. Chem. 121 (2009), 9445.

[9] S.N. Varanakkottu, S.D. George, T. Baier, S. Hardt, M. Ewald, and M. Biesalski: Angew. Chem. Int. Ed. (2013), 7291.

[10] S. Ma, Y. Kuwahara, H. Nagano, N. Hatae, T. Ogata, S.N. Kim, and S. Kurihara: Mol. Cryst. Liq. Crys. 601 (2014), 126.

[11] S. Kurihara, K. Ohta, T. Oda, R. Izumi, Y. Kuwahara, T. Ogata, S.N. Kim: Sci. Rep. 3 (2003), 2167.

[12] V Luzzati, H Mustacchi, A Skoulios: Nature 180 (1957), 600.

[13] F. B. ROSEVEAR:J. Am. Oil. Chem. Soc. 31 (1954), 628.

[14] C.E. Fowler, W. Shenton, G. Stubbs, and S. Mann: Adv. Mater. 13 (2001), 1266.

[15] K. Kanie, J. Sekiguchi, X. Zeng, G. Ungar and A. Muramatsu: Chem. Commun. 47 (2011), 6885.

[16] V. Ramesh, H.S. Chien and M.M.Labes: J. Phys. Chem. 91 (1987), 5937.

[17] H.M. Dhammika, Bandara and S.C. Burdett: Chem. Soc. Rev. 41 (2012), 1809. 